\title{
Artificial Intelligence and Military Applications: Innovations, Cybersecurity Challenges \& Open Research Areas
}

\author{
Priyanka Jayakumar \\ School of Computer Science and Engineering \\ Taylor's University \\ Selangor, Malaysia \\ priyankas.jayakumar@sd.taylors.edu.my
}

\author{
Sarfraz Nawaz Brohi \\ School of Information Technology \\ Monash University \\ Selangor, Malaysia \\ sarfraz.nawazbrohi@monash.edu
}

\author{
NZ Jhanjhi \\ School of Computer Science and Engineering \\ Taylor's University \\ Selangor, Malaysia \\ noorzaman.jhanjhi@taylors.edu.my
}

\begin{abstract}
Over the years, Artificial Intelligence (AI) has seen a steady progress in development and evolution that can aid many sectors. Today, AI plays an essential role in the Fourth Industrial Revolution, and it is making its way into the military sector as well. Many countries are actively looking into AI military technology. Some of these innovations include image recognition, text analysis, Self-driving vehicles (SDV), gaming, robotic process automation (RPA) \& Robotic and Autonomous Systems (RAS), and Autonomous Weapons Systems (AWS). In this research, we discuss the advantages of each innovation mentioned and analyze the many different cybersecurity threats that awaken due to military artificial intelligence systems. We then discuss the open research areas to better improve the current state of military artificial intelligence applications in terms of ethics, system security, proper strategy plan, accepted responsibility matrix, and introduction of relevant laws.
\end{abstract}

\section{Keywords-Artificial Intelligence, Military technology, Robotic and Autonomous systems, Autonomous weapons systems}

\section{INTRODUCTION}

Artificial Intelligence (AI) is the term coined to refer to the ability of a digital computer or computer-controlled robot to carry out tasks that are normally done by intelligent beings. When the development of a system's processes has human characteristics (ability to reason, discover meaning, generalize, or learn from the past), then it is said that that system has incorporated artificial intelligence [1-2].

There are many advantages that AI bring to mankind. Some of these advantages include automation, smart decision making, medical revolution, boosted customer experience, business continuity, handling repetitive tasks, reducing manual errors, research and data analysis, solving difficult problems, improved business efficiency, and many more. Automation is one of the most celebrated benefit of AI because it leads to improved production rates and productivity as well as makes way for more efficient use of raw materials, improved product quality, reduced lead times, and superior safety across various sectors/industries [3-4].

When this brilliant technology first emerged, researchers and professional experts could not get enough of it because it opened up endless opportunities across different sectors. For years, many AI-centric research and system developments were in progress but in today's reality, many of these theoretical opportunities have already become successful and useful AI applications. Some of the useful AI applications today are autonomous vehicles, called Self-driving Vehicles (SDV), that enables blind or physically impaired people to drive anywhere without 
help or supervision; implementation of Robotic Process Automation (RPA) in which simple rule-based activities are replicated to increase efficiency and accuracy, safe cost as well as save humans from dull and monotonous jobs; the usage of chatbots have not only helped customer service representatives to reduce their workload but also provided immediate responses to queries with lower errors and service time, enhanced customer engagement with the ability to handle simple transactions; AI incorporated payment services can make hassle-free, authorized payments and also arrange for shipping to homes when running low on supplies. [5-6]

Just like a coin, artificial intelligence has two sides. The only aspect AI lacks is emotional intelligence. If only AI systems can think and feel like humans do, then not only could they prove highly useful for mankind but they can potentially also be the most destructive technology for humankind. As mentioned before, today's world is witnessing the power of AI, especially in the military sector. AI is being a huge relief for military personnel because it eases a major part of their day-to-day operations as well as long-term missions. However, being too dependent on $\mathrm{AI}$ and wrongful usage of $\mathrm{AI}$ in military technology is possible and extremely dangerous. AI in military technology poses 3 types of problems - ethical, operational, and strategic risks. Ethical risks evolve around the line between right and wrong actions, operational risks are where the dependability, delicacy, and security of AI systems come into the picture, and strategic risks are the problems AI might invite or escalate by simply being implemented in military technology. [7-8].

\section{AI Military TeChNology InNOVAtions}

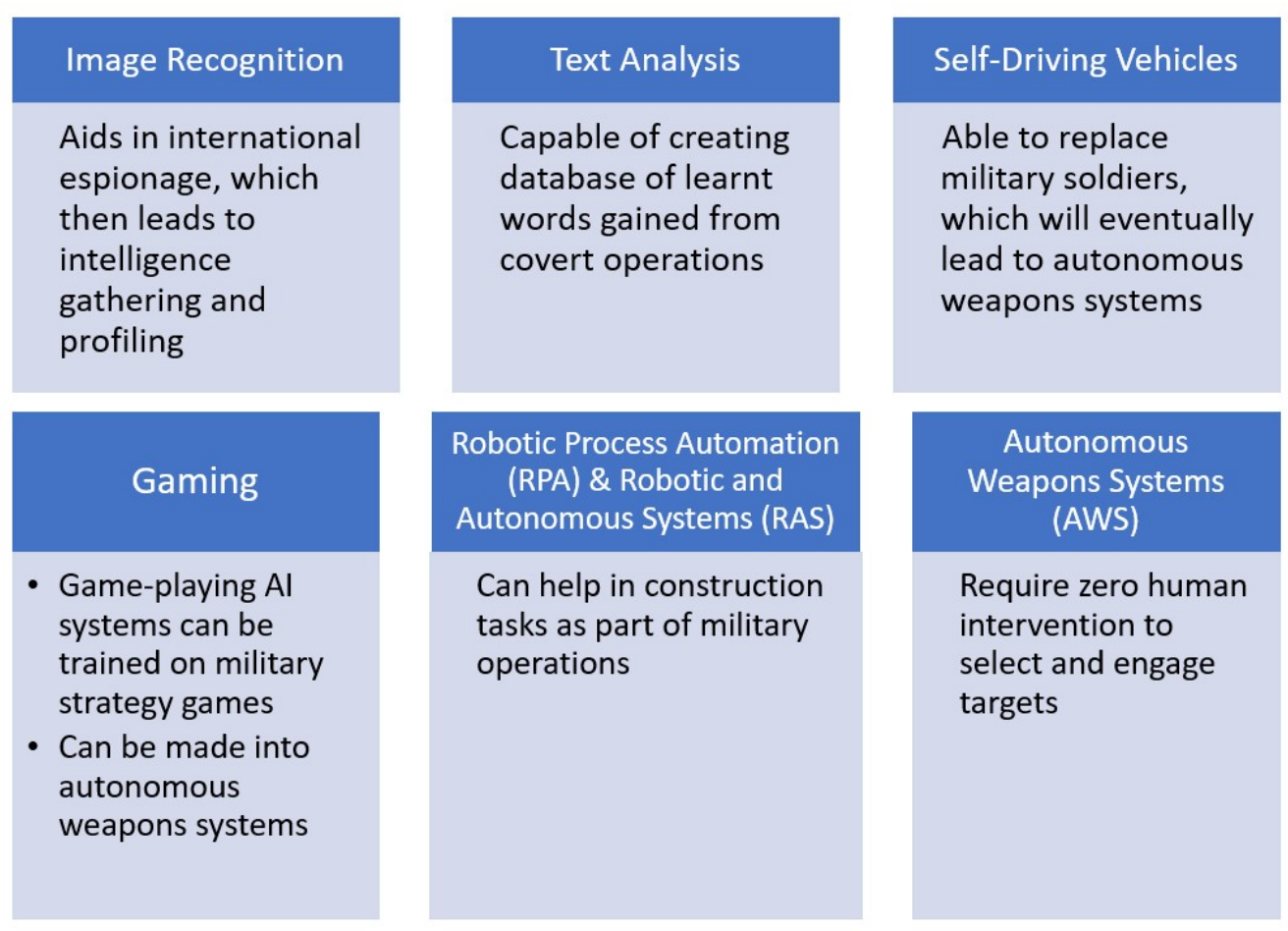

Figure 1: Taxonomy of AI innovations in the military sector

\section{A. Image Recognition}

Moravec's paradox is an important finding in the AI domain that says tasks that are easy and effortless for human beings are complex and difficult for computers - and vice versa [9]. Image recognition is one such phenomenon. Thanks to the evolution of deep neural networks, computers have now gained the ability to 
recognise images and detect objects and in some instances, they can perform these tasks with a higher accuracy than humans can. Today, researchers and academicians are exploring randomly wired neural networks with different models to deduce which is the best way to go about improved image recognition [10]. Powered by deep learning, the precision of computers to recognise images is fine-tuned by exposing them to high volumes of labelled images. Due to this exposure, not only can computers identify images as humans do, but they can also pick up even the most subtle facial expressions that leads to biometric identification through facial features. When computers can analyse facial expressions, naturally they will be able to associate them with emotions [11]. From a military view, image recognition can aid in international espionage, which then leads to intelligence gathering and profiling. Combining image recognition with military weapons can ease the process of identifying and executing targets, especially if the weapons themselves have AI integrated in them.

\section{B. Text Analysis}

To understand text analysis, knowing the difference between structured and unstructured data is vital. Structured data refers to data that has a set format like whole numbers, statistics, spreadsheet and databases while unstructured data revolves around images, audio, video, and of course, text. The unmanned process of making sense and grouping unstructured data with AI-driven machine learning is called text analysis. The main purpose of text analysis is to make data-driven means a possibility, by deriving machine-readable information from unstructured data [12-13]. Some example of text analysis activities includes text categorization, text clustering, sentiment analysis, document summarization, and thematic analysis [14]. In military context, a system that can perform text analysis can create an entire database of learnt words gained from covert operations for future operations with better clarity and response. Spying and understanding plans of the enemy could not be easier. The military would also be saving manpower on this front, seeing how text analysis is an automated process.

\section{Self-Driving Vehicles}

The world has seen the evolution of self-driving vehicles. This includes cars on land and planes in the air. The main purpose of SDVs is to transport people or objects to a pre-established destination without the intervention of human beings [15]. In the context of self-driving cars, even blind people can travel on their own without depending on others while self-flying aircrafts takes the burden off pilots from over-straining themselves since statistics show that the volume of passengers and flights outweighs the number of qualified pilots available in the aviation industry [16]. Most SDVs are supervised autonomous systems, which refers to systems that require human intervention to operate properly. Even with human supervision, this particular application in AI is controversial due to safety concerns since human lives is at stake [17-18]. However, despite the safety concerns, the steady development and research in safety measures in SDVs are slowly gaining the trust of drivers and passengers. From a military point of view, they could easily use this AI application to replace military soldiers with these autonomous systems, which will eventually lead them to autonomous weapons systems as well. Instead of physically being present in a tank, battlefield or any situation that can put lives at risk, soldiers only need to learn how to control and supervise SDVs to carry out intended tasks, as simple as delivering supplies to troops in different areas.

\section{Gaming}

The ability to play games is a great way to measure the advancement of AI ever since its inception. In 2019, AlphaStar - built by Google's AI firm Deepmind - defeated the best of human players at a popular online strategy game called StarCraft II and emerged as the grandmaster as well as finding itself within the top $0.15 \%$ of the region's 90,000 players. This huge leap in game-playing AI systems left researchers stunned because they did not expect AI to master this area so soon, especially when a game as complex and fast-paced as StarCraft II requires professional players to make more than 300 actions per minute [19]. A technique called reinforcement learning plays a crucial role in these systems. Reinforcement Learning (RL) is one of the three 
basic Machine Learning (ML) paradigms that trains ML models to make an order of decisions [20]. RL is comprised of two main components: the environment (the game) and the agent (our AI avatar that is driven by deep neural network). RL revolves around a reward system in which the environment gives a reward to the agent based on how well it has decided to carry out an action. The ultimate purpose of the agent is to learn what actions maximize the reward, given every possible state [21-22]. Each time a game-playing AI system goes through RL, it becomes better since it pushes itself to improve based on this reward system. In military context, game-playing AI systems can be trained on war games or tower defense games where military strategy is emphasized. With RL, these well-versed game-playing AI systems can be made into autonomous weapons systems, where little to no human intervention is required, because these AI systems are more than capable of handling themselves in a warzone.

\section{E. Robotic Process Automation \& Robotic and Autonomous Systems}

Automation and robotics soon became necessities when digital transformation started evolving and at-present, Robotic Process Automation has gotten corporate attention pertaining to automation initiatives [23]. RPA is a hypernym for tools that work on the user interface of other computer systems, as humans do. It allows for computer systems or "robots" to imitate and merge the activities of a human interacting within digital systems to carry out a business process. RPA tools' main objective is to save employees from the burden of repetitive, simple, and monotonous tasks [24-25]. RPA's full potential was brought to life with the birth of Robotic and Autonomous Systems. RAS refers to any intelligent digital system or a physical "robot" that can carry out tasks without human interference [26]. Agriculture, healthcare, manufacturing, logistics, production, and transportation are some of the many application areas of RAS. Researchers predict that the military can benefit from RAS in the civil construction sector since ground-based military forces are often assigned to perform construction tasks as part of military operations, where these tasks can be partially or fully aided by the employment of RAS technologies [27]. In another research paper, researchers have come up a four-tier taxonomy of military functions that could be carried out utilizing RAS-solutions, as shown in Figure 2 [28].

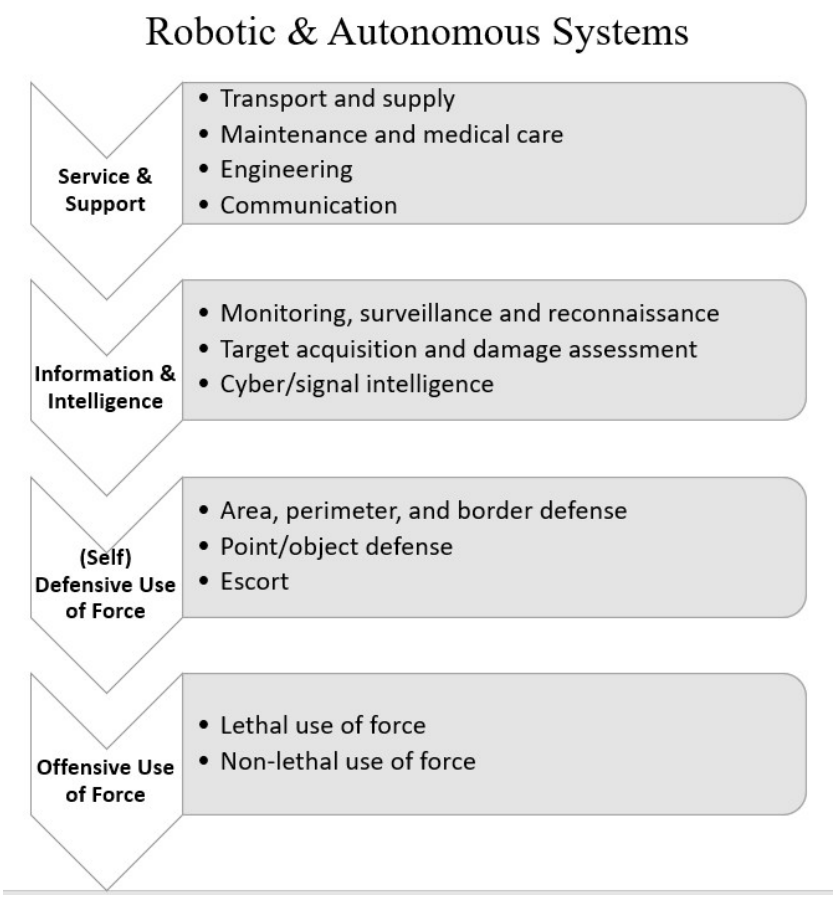

Figure 2: Taxonomy of military functions for RAS 


\section{F. Autonomous Weapons Systems}

Currently, when it comes to AI innovations in military, Autonomous Weapons Systems (AWS) is the pinnacle of the conversation. Fully autonomous weapons, otherwise known as lethal autonomous weapons systems (LAWS) or killer robots, are weapon systems that are dependent on sensor inputs that results from sensor processing to select and engage targets - with zero human intervention [29]. While the existence of human robots is highly unlikely today, the precursor weapons systems that do exist today is proof of what to expect in the near future. The development, application and implementation of AWS is riddled with huge impacts that can effortlessly tilt the fragile balance that is preserving world peace. Some of the consequences of AWS implementation includes machines making the decision of whether or not to take a life, the lack of human element could make the decision to go to war look more favoured, the occurrence of catastrophic blunders that could initiate or worsen tension is highly likely, the lack of human judgement to differentiate civilians from combatants and strictly obey the laws of warfare, and existence of responsibility gaps with regards to unlawful acts caused by AWS [30 - 32]. This AI application in military can only cause more damage than it brings advantage since it does not only affect military personnel of involved countries but also the people and ultimately world peace, as we know it.

\section{CYBERSECURITY THREATS DUE TO MILITARY ARTIFICIAL INTELLIGENCE}

Many threats are affiliated to the usage of artificial intelligence in upcoming, advanced technology. With regards to military artificial intelligence, the seriousness of threats is significant. The fact that most military systems are connected to the Internet does not help reduce the cyber threat landscape, either. Adversaries can easily tap into these automated systems, especially if they have the smallest of vulnerabilities in their design or coding or unpatched vulnerabilities. For example, some of the military artificial intelligence innovations discussed in Section 2 are image recognition, text analysis, and self-driving vehicles. An infected image recognition system will result in the AI-powered system to recognize images wrongly, that will trigger wrong actions for an incorrectly identified image and this, can cause lives. Poorly analyzed text can end up in corrupt data that would either be useless for covert operations or will lead military personnel in building a plan that is based on invalid data and drive the entire operation in a wrong path altogether. As for self-driving vehicles, if not properly configured or trained, then this can result in fatal consequences. To proof that, the world has already witnessed the death of 49-year-old Elaine Herzberg in Arizona - making it the world's first recorded pedestrian fatality involving a self-driving vehicle [33 - 37]. Other than these AI applications, there are other cybersecurity threats that can negatively implicate military artificial intelligence such as staged payloads, AI-integrated malware, and converged risks. With the malicious intent of evading machine learning (ML) algorithms, malware authors are creating malware that has a larger portion of innocent, benign code and small portion of payload. To take it a step further, malware is now being built with artificial intelligence that enables them to constantly change codes, making it possible for bypass of facial security and spam filters, successful fake voice commands and evasion of anomaly detection engines and security defenses. Besides that, a lot of operations today are governed and controlled by different technologies. Operational technology (OT) and information technology (IT) are no longer separate networks and so now, the cyber security risks are high. Data breach in military AI systems due to cyber security threats can jeopardize an entire country's safety [38 - 40]. Cybersecurity is a critical issue overall for all the domains, [41-46] including the industry 4.0, bigdata application, smart cities, military applications, etc. Cybersecurity threats are several in number however, most of the literature group them in top ten threads [47-49] which have more impact on different domain, and researchers proposed different secure frameworks as well. 


\section{OPEN RESEARCH AREAS IN MILITARY ARTIFICIAL INTELLIGENCE}

As mentioned before, the development of artificial intelligence in the military sector creates ethical, operational, and strategic risks. Leaving machines to decide whether to kill or not and using AI to do physically non-violent but privacy-violating activities under the name of national security are among the top two ethical issues. Even though AI has come a long way throughout these years, it is not perfect and therefore, capable of making mistakes. If AI is going to be incorporated in the military sector, intelligent systems need to be completely reliable since mistakes are a luxury in terms of operations. Besides that, proper military strategizing is vital to avoid unnecessary conflicts. Knowing when and what type of AI system to deploy, the necessity and suitability of an AI system to handle a situation, and the actions an AI system is allowed to perform are among the important questions that need to be answered before proceeding with a strategy plan. Open research areas include looking into perfecting existing AI innovations to minimize or completely get rid of human safety concerns, coming up with clear and accepted responsibility matrix frameworks and introduction of relevant laws should AI-integrated systems lead to fatal incidents, and amicably concluding negotiations on a new international treaty to retain meaningful human control over AWS.

\section{REFERENCES}

[1] B.J. Copeland, B., 2020. Artificial Intelligence| Definition, Examples, And Applications. [online] Encyclopedia Britannica. Available at: <https://www.britannica.com/technology/artificial-intelligence $>$ [Accessed 9 January 2021].

[2] IBM Cloud Education. (2021). What is Artificial Intelligence (AI)?. Retrieved 9 January 2021, from https://www.ibm.com/cloud/learn/what-is-artificial-intelligence

[3] 10xds.com. 2020. Top 10 Benefits Of Artificial Intelligence (AI). [online] Available at: $<$ https://www.10xds.com/blog/benefits-of-artificial-intelligence-ai/> [Accessed 9 January 2021].

[4] Hartwig, B. (2021). Benefits of Artificial Intelligence. Retrieved 21 January 2021, from https://hackr.io/blog/benefitsof-artificial-intelligence

[5] Qasmi, M. (2021). 15 Business Opportunities Enabled by Artificial Intelligence - MobileLIVE Inc. Retrieved 21 January 2021, from https://perspectives.mobilelive.ca/blog/15-artificial-intelligence-opportunities

[6] Adixon, R. (2021). Artificial Intelligence Opportunities \& Challenges in Businesses. Retrieved 21 January 2021, from https://towardsdatascience.com/artificial-intelligence-opportunities-challenges-in-businesses-ede2e96ae935

[7] Morgan, F., Boudreaux, B., Lohn, A., Ashby, M., Curriden, C., Klima, K., \& Grossman, D. (2020). Military Applications of Artificial Intelligence: Ethical Concerns in an Uncertain World (pp. 24 - 48). Santa Monica, California: RAND Corporation.

[8] Sisson, M., Spindel, J., Scharre, P., \& Kozyulin, V. (2021). The Militarization of Artificial Intelligence. United Nations, Office For Disarmament Affairs, 20 - 22. Retrieved from https://www.un.org/disarmament/themilitarization-of-artificial-intelligence/

[9] Tombs, R. (2021). Moravec's Paradox - Who, What, When and Where and let's also ask Why ?. Retrieved 21 January 2021, from https://technicalunemployment.com/2020/07/03/moravecs-paradox-who-what-when-and-where-and-letsalso-ask-why/

[10] Xie, S., Kirillov, A., Girshick, R., \& He, K. (2019). Exploring Randomly Wired Neural Networks for Image Recognition. International Conference On Computer Vision. Retrieved from https://arxiv.org/abs/1904.01569

[11] Svenmarck, P., Luotsinen, L., Nilsson, M., \& Schubert, J. (2018). Possibilities and Challenges for Artificial Intelligence in Military Applications. Swedish Defence Research Agency. Retrieved from https://www.researchgate.net/publication/326774966_Possibilities_and_Challenges_for_Artificial_Intelligence_in_ Military_Applications

[12] MonkeyLearn. (2020). Text Analysis. Retrieved 21 January 2021, from https://monkeylearn.com/text-analysis/

[13] Ontotext KnowledgeHub. (2021). What is Text Analysis? | Ontotext Fundamentals Series. Retrieved 21 January 2021, from https://www.ontotext.com/knowledgehub/fundamentals/text-analysis

[14] Library of The University of Queensland. (2021). Library Guides: Text mining \& text analysis: Text mining/analysis activities. Retrieved 21 January 2021, from https://guides.library.uq.edu.au/research-techniques/text-mininganalysis/text-analysis-activities

[15] Zhao, J., Liang, B., \& Chen, Q. (2018). Article The key technology toward the self-driving car. International Journal Of Intelligent Unmanned Systems, Vol. 6 No. 1. doi: 10.1108/IJIUS-08-2017-0008

[16] Talley, S. (2020). Public Acceptance of AI Technology in Self-Flying Aircraft. Journal Of Aviation/Aerospace Education \& Research, 29(1). doi: 10.15394/jaaer.2020.1822

[17] Nyholm, S. (2017). Attributing Agency to Automated Systems: Reflections on Human-Robot Collaborations and Responsibility-Loci. Science And Engineering Ethics, 24(4), 1201-1219. doi: 10.1007/s11948-017-9943-x

[18] Nyholm, S. (2018). The ethics of crashes with self-driving cars: A roadmap, II. Philosophy Compass, 13(7), e12506. doi: $10.1111 / \mathrm{phc} 3.12506$ 
[19] Garisto, D. (2019). Google AI beats top human players at strategy game StarCraft II. Retrieved 24 January 2021 , from https://www.nature.com/articles/d41586-019-03298-6

[20] Osiński, B., \& Budek, K. (2018). What is reinforcement learning? The complete guide - deepsense.ai. Retrieved 24 January 2021, from https://deepsense.ai/what-is-reinforcement-learning-the-complete-guide/).

[21] Comi, M. (2018). How to teach an AI to play Games: Deep Reinforcement Learning. Retrieved 24 January 2021, from https://towardsdatascience.com/how-to-teach-an-ai-to-play-games-deep-reinforcement-learning-28f9b920440a

[22] Goldwaser, A., \& Thielscher, M. (2020). Deep Reinforcement Learning for General Game Playing. Proceedings of the AAAI Conference on Artificial Intelligence, 34(02), 1701-1708. https://doi.org/10.1609/aaai.v34i02.5533

[23] Hofmann, P., Samp, C., \& Urbach, N. (2019). Robotic process automation. Electronic Markets, 30(1), 99-106. doi: $10.1007 / \mathrm{s} 12525-019-00365-8$

[24] UiPath Inc. (2021). What is Robotic Process Automation - RPA Software | UiPath. Retrieved 25 January 2021, from https://www.uipath.com/rpa/robotic-process-automation

[25] Van der Aalst, W., Bichler, M., \& Heinzl, A. (2018). Robotic Process Automation. Business \& Information Systems Engineering, 60(4), 269-272. doi: 10.1007/s12599-018-0542-4

[26] Bekey, G. A. (2017). Autonomous Robots: From Biological Inspiration to Implementation and Control (Intelligent Robotics and Autonomous Agents series) (Reprint ed.). Bradford Books.

[27] Ha, Q., Yen, L., \& Balaguer, C. (2018, July). Earthmoving Construction Automation with Military Applications: Past, Present and Future. Proceedings of the 35th International Symposium on Automation and Robotics in Construction (ISARC). https://doi.org/10.22260/isarc2018/0164

[28] Torossian, B., Bekkers, F., Sweijs, T., Roelen, M., Hristov, A., \& Atalla, S. (2020, February). The Military Applicability of Robotic and Autonomous Systems. The Hague Centre for Strategic Studies. https://hcss.nl/report/military-applicability-robotic-and-autonomous-systems

[29] Docherty, B. (2019, November). Key Elements of a Treaty on Fully Autonomous Weapons. Stop Killer Robots. https://www.stopkillerrobots.org/wp-content/uploads/2020/04/Key-Elements-of-a-Treaty-on-Fully-AutonomousWeaponsvAccessible.pdf

[30] The Campaign To Stop Killer Robots. (2020). The Campaign To Stop Killer Robots. Stop Killer Robots. https://www.stopkillerrobots.org/learn/

[31] Klare, M. T. (2019, March). Autonomous Weapons Systems and the Laws of War. Arms Control Association. https://www.armscontrol.org/act/2019-03/features/autonomous-weapons-systems-laws-war

[32] Anderson, K., \& Waxman, M. C. (2017). Debating Autonomous Weapon Systems, Their Ethics, and Their Regulation Under International Law. Oxford Handbook of Law, Regulation, and Technology, $1097-1117$. https://papers.ssrn.com/sol3/papers.cfm?abstract_id=2978359

[33] Coicheci, S., \& Filip, I. (2021). Self-driving vehicles: current status of development and technical challenges to overcome. Retrieved 5 July 2021, from https://ieeexplore.ieee.org/abstract/document/9118809

[34] J.Hawkins, A. (2021). The world's first robot car death was the result of human error - and it can happen again. Retrieved 5 July 2021, from https://www.theverge.com/2019/11/20/20973971/uber-self-driving-car-crashinvestigation-human-error-results

[35] Combs, T., Sandt, L., Clamann, M., \& McDonald, N. (2019). Automated Vehicles and Pedestrian Safety: Exploring the Promise and Limits of Pedestrian Detection. American Journal Of Preventive Medicine, 56(1), 1-7. doi: 10.1016/j.amepre.2018.06.024

[36] Shariff, A., Bonnefon, J., \& Rahwan, I. (2021). How safe is safe enough? Psychological mechanisms underlying extreme safety demands for self-driving cars. Transportation Research Part C: Emerging Technologies, 126, 103069. doi: $10.1016 / \mathrm{j} . \operatorname{trc} .2021 .103069$

[37] Schmelzer, R. (2021). What Happens When Self-Driving Cars Kill People?. Retrieved 5 July 2021, from https://www.forbes.com/sites/cognitiveworld/2019/09/26/what-happens-with-self-driving-cars-killpeople/?sh $=61 \mathrm{addb} 5 \mathrm{~b} 405 \mathrm{c}$

[38] GlobalData Thematic Research. (2021). Cybersecurity in Defence: AI Technology Trends. Retrieved 5 July 2021, from https://www.army-technology.com/comment/cybersecurity-defence-ai-technology-trends

[39] Ahmad, J., Amir, S., \& Ahmad, F. (2021). Artificial intelligence and its prospective use in armed forces. Electronic Research Journal Of Engineering, Computer And Applied Sciences, 1, 100-117. doi: http://erjsciences.info/wpcontent/uploads/2020/11/10.-AI-19.pdf

[40] Khisamova, Z., Begishev, I., \& Sidorenko, E. (2021). Artificial Intelligence and Problems of Ensuring Cyber Security. International Journal Of Cyber Criminology 2019, 13(2), 564-577. doi: 10.5281/zenodo.3709267

[41] Humayun, M. (2021). Industry 4.0 and Cyber Security Issues and Challenges. Turkish Journal of Computer and Mathematics Education (TURCOMAT), 12(10), 2957-2971.

[42] N. Jhanjhi, M. Humayun and S. N. Almuayqil, "Cyber security and privacy issues in industrial internet of things," Computer Systems Science and Engineering, vol. 37, no.3, pp. 361-380, 2021.

[43] D. K. Alferidah and N. Jhanjhi, "Cybersecurity Impact over Bigdata and IoT Growth," 2020 International Conference on Computational Intelligence (ICCI), 2020, pp. 103-108, doi: 10.1109/ICCI51257.2020.9247722

[44] Almrezeq, N. (2021). Cyber Security Attacks and Challenges in Saudi Arabia during COVID-19. Turkish Journal of Computer and Mathematics Education (TURCOMAT), 12(10), 2982-2991.

[45] Humayun, M., Niazi, M., Jhanjhi, N. et al. Cyber Security Threats and Vulnerabilities: A Systematic Mapping Study. Arab J Sci Eng 45, 31713189 (2020). https://doi.org/10.1007/s13369-019-04319-2

[46] B. Hamid, N. Jhanjhi, M. Humayun, A. Khan and A. Alsayat, "Cyber Security Issues and Challenges for Smart Cities: A survey," 2019 13th International Conference on Mathematics, Actuarial Science, Computer Science and Statistics (MACS), 2019, pp. 1-7, doi: 10.1109/MACS48846.2019.9024768. 
[47] Khan, Navid Ali; Brohi, Sarfraz Nawaz; Zaman, Noor (2020): Ten Deadly Cyber Security Threats Amid COVID-19 Pandemic. TechRxiv. Preprint. https://doi.org/10.36227/techrxiv.12278792.v1

[48] Zaman, N., \& Ahmad, M. (2017). Towards the evaluation of authentication protocols for mobile command and control unit in healthcare. Journal of Medical Imaging and Health Informatics, 7(3), 739-742.

[49] Ponnusamy, V., Jhanjhi, N. Z., \& Humayun, M. (2020). Fostering Public-Private Partnership: Between Governments and Technologists in Developing National Cybersecurity Framework. In V. Ponnusamy, K. Rafique, \& N. Zaman (Ed.), Employing Recent Technologies for Improved Digital Governance (pp. 237-255). IGI Global. http://doi:10.4018/978-1-7998-1851-9.ch012 